in particular in relation to people with more than two of these experiences and for health outcomes other than infections. This situation is exacerbated by the under-ascertainment of these populations in routine information sources on population health needs, such as surveys and censuses. In many countries, administrative data are available which could help address these knowledge gaps. We describe the creation and characteristics of a novel virtual cohort using cross-sectoral linkage of administrative datasets, in order to inform policy and practice responses to these co-occurring issues.

Methods Individual-level data from local authority homelessness services (HL), opioid substitution therapy dispensing (OST), and a psychosis case register (PSY) in Glasgow, Scotland between 2011-15 were confidentially linked to National Health Service records, using a mix of probabilistic and deterministic linkage. A de-identified dataset was made available to researchers through a secure analysis platform. Demographic characteristics associated with different exposure combinations were analysed using descriptive statistics.

Results Linkage created a cohort of 24,767 unique individuals with any one of the experiences of interest between 2011-15. Preliminary results suggest that $89.2 \%$ of the cohort had one experience; $10.6 \%$ two; and $0.2 \%$ all three. The most common combination was HL \& OST ( $\mathrm{O}=2,150 ; 8.7 \%)$, with other combinations much less frequent (HL \& PSY, $n=279$, $1.1 \%$; OST \& PSY, $\mathrm{n}=188,0.8 \%$; HL \& OST \& PSY, $\mathrm{n}=51$, $0.2 \%)$. The odds of male gender increased with number of exposures (2 exposures, OR 2.1, 95\% CI 1.9-2.2; 3 exposures, OR 4.1, 95\% CI 2.3-7.2), but there was little difference in age. Work is ongoing to incorporate into the cohort additional datasets on criminal justice involvement.

Discussion Administrative data linkage is a feasible approach to understanding the health of people affected by multiple exclusionary processes, addressing problems of recruitment and retention affecting traditional cohort studies in this field. As well as improving the validity of descriptive epidemiology for these populations, this study offers a foundation for evaluating future policy or service interventions. In order for the benefits of administrative data research to be realised, robust and timely governance and linkage processes are required.

\section{OP21 INEQUALITIES IN THE PREVALENCE AND DEVELOPMENT OF MULTIMORBIDITY ACROSS ADULTHOOD: FINDINGS FROM THE 1946 NATIONAL SURVEY OF HEALTH \& DEVELOPMENT}

\footnotetext{
${ }^{1,2}$ AR Khanolkar*, ${ }^{1} \mathrm{~N}$ Chaturvedi, ${ }^{1} \mathrm{D}$ Davis, ${ }^{1} \mathrm{~A}$ Hughes, ${ }^{1} \mathrm{M}$ Richards, ${ }^{3} \mathrm{~V}$ Kuan Po Ai, ${ }^{4} \mathrm{D}$ Bann, ${ }^{1,4} \mathrm{P}$ Patalay. ${ }^{1} \mathrm{MRC}$ Unit for Lifelong Health and Ageing at UCL, University College London, London, UK; ${ }^{2}$ Institute for Environmental Medicine, Karolinska Institutet, Stockholm, Sweden; ${ }^{3}$ Institute of Health Informatics, University College London, London, UK; ${ }^{4}$ Centre for Longitudinal Studies, University College London, London, UK
}

\subsection{6/jech-2020-SSMabstracts.21}

Background With increasing life expectancy and aging populations, the prevalence of multimorbidity (two or more conditions in a person) is rising. Multimorbidity is progressively more common in older age, socioeconomically disadvantaged groups and associated with increased mortality and morbidity. Most studies on multimorbidity to date are cross-sectional in design or with limited follow-up.

We studied the development of multimorbidity across adulthood and early old age in a nationally representative birth cohort study.
Methods This study analysed data from the 1946 National Survey of Health and Development (NSHD). The analysis sample included participants who attended the age 36 assessment in 1982 and any one of the follow-up assessments (ages 43, 53, $63 \& 69 ; \mathrm{N}=3,723,51 \%$ males). Information on 18 common conditions was based on a combination of selfreport, prescribed medications and health records.

Conditions included diabetes, dyslipidaemia, hypertension, obesity, coronary heart disease, stroke, cancer, anaemia, respiratory-, kidney-, gastro intestinal-, skin-disorders, arthritis, Parkinson's disease, epilepsy, depression \& psychosis.

For all participants, we calculated a multimorbidity score at each age indicating the number of conditions accumulated over time and was the outcome of interest. Linear-spline mixed-effects modelling was used to study the population-average accumulation of conditions over time in different periods. We also assessed sex and socioeconomic differences in longitudinal trajectories of multimorbidity across the five ages (18,615 data points, mean: 5 data points/participant).

Childhood social class and adulthood educational level were used as socioeconomic indicators. Missing data was addressed using multiple imputation.

Results Proportion of participants with no conditions decreased progressively from $52 \%$ at age 36 to $7 \%$ at age 69 . Multimorbidity (the number of conditions) increased progressively across all 4 periods $(0.55,95 \%$ CI $[0.5,0.6]$ for $1982-$ $89,0.63[0.58,0.7]$ for $1989-99,0.70$ [0.63, 0.78] for 1999-09 and 1.15 [1.04, 1.25] for 2009-15). Disadvantaged social class in childhood was associated with marginally increased multimorbidity in adulthood $(0.08$ [0.01, 0.15] for skilled/unskilled and 0.07 [-0.01, 0.15] for manual groups compared to professional/intermediate group). Higher educational attainment was associated with decreased risk for multimorbidity $(-0.09[-0.2,0.01]$ for university degree and -0.13 $[-0.2,-0.06]$ for General Certificate of Education (GCE) compared to those without education). Estimates for childhood social class were attenuated and no longer significant when adjusted for educational level. Women had marginally higher risk for multimorbidity compared to men $(0.15$ [0.09-0.2]).

Conclusion Multimorbidity increases progressively with age, with the socioeconomically more disadvantaged having greater multimorbidity.

\section{OP22 UNDERSTANDING PATHWAYS TO HEALTH INEQUALITIES IN CYSTIC FIBROSIS - A CAUSAL MEDIATION ANALYSIS USING UK REGISTRY DATA}

${ }^{1}$ DK Schlüter*, ${ }^{2} R$ Keogh, ${ }^{3} S$ Agbla, ${ }^{4} R$ Daniel, ${ }^{1} D$ Taylor-Robinson. ${ }^{1}$ Public Health and Policy, University of Liverpool, Liverpool, UK; ${ }^{2}$ Medical Statistics, London School of Hygiene and Tropical Medicine, London, UK; ${ }^{3}$ Biostatistics, University of Liverpool, Liverpool, UK; ${ }^{4}$ School of Medicine, Cardiff University, Cardiff, UK

\subsection{6/jech-2020-SSMabstracts.22}

Background Cystic fibrosis (CF) is an inherited, progressive condition affecting over 10000 individuals in the UK. Symptoms of CF include poor growth, lung infection, poor lung function and reduced survival. Outcomes are worse for people growing up in disadvantaged circumstances, but it is not clear to what extent inequalities in early growth can explain inequalities in later lung function and survival. Our aim was to assess how the association between socioeconomic circumstances (SECs) and subsequent lung function measured around age 6 is mediated by weight trajectory in early childhood. 